\title{
Investigando a Influência da Distribuição Espacial de Mosquitos na Propagação de Doenças via Autômato Celular
}

\author{
Julio Cesar de Azevedo Dias ${ }^{1}$, Luiz Henrique Alves Monteiro ${ }^{1,2}$ \\ ${ }^{1}$ Programa de Pós-graduação em Engenharia Elétrica e Computação da Universidade \\ Presbiteriana Mackenzie, São Paulo, SP \\ ${ }^{2}$ Escola Politécnica da Universidade de São Paulo, São Paulo, SP \\ julio.dias@gmail.com, luizm@mackenzie.br, luizm@usp.br
}

\begin{abstract}
In Brazil, contagious diseases transmitted by vectors are a serious public health problem. In this work, it is investigated how the spatial distribution of mosquitoes affects the spreading of diseases such as chikungunya. This investigation is based on a SIR-type model. The model is formulated in terms of a cellular automaton, in which each cell of the lattice corresponds to an individual of the host population. At each time step, each individual is in one of three states: susceptible (S), infected (I), or recovered (R). Four kinds of spatial distribution of mosquitoes are considered. The results of numerical simulations are discussed from an epidemiological point of view.
\end{abstract}

Resumo. No Brasil, doenças contagiosas transmitidas por vetores são um problema grave de saúde pública. Neste trabalho, investiga-se como a distribuição espacial de mosquitos afeta a propagação de doenças, como chicungunha. Essa investigação é baseada num modelo do tipo SIR. O modelo é formulado em termos de um autômato celular, em que cada célula do reticulado corresponde a um indivíduo da população hospedeira. A cada passo de tempo, cada indivíduo está em um de três estados: suscetivel (S), infectado (I), ou recuperado (R). Quatro tipos de distribuição espacial de mosquitos são considerados. Os resultados de simulações numéricas são discutidos de um ponto de vista epidemiológico.

\section{Introdução}

Muitos estudos epidemiológicos têm como objetivo prever como uma doença contagiosa se propaga, a fim de se elaborarem estratégias que possam eliminar ou, ao menos, controlar essa propagação [Anderson and May, 1991; Friis, 2009; Hethcote, 2000]. No Brasil, doenças transmitidas por vetores, em particular, por mosquitos, têm se tornado um grave problema de saúde pública. Nas últimas décadas, o Brasil tem padecido de epidemias recorrentes de dengue [Ministério da Saúde, 2017a,b]. Mais recentemente, chikungunya (ou chicungunha) e zika têm causado preocupação [Ministério da Saúde, 2017a,b]. E até a febre amarela, supostamente erradicada de centros urbanos, voltou ao noticiário após provocar dezenas de mortes no início de 2017 [Ministério da Saúde, 2017c]. Por isso, é relevante investigar como a distribuição espacial de mosquitos influencia a disseminação de doenças como aquelas mencionadas neste parágrafo. 
Quando se deseja estudar a propagação de doenças levando em conta a região em que a população hospedeira vive, usam-se, em geral, modelos escritos ou em termos de equações a derivadas parciais [Murray, 2011] ou em termos de autômatos celulares [Wolfram, 1994]. Equações a derivadas parciais possibilitam a dedução de relações analíticas que colaboram para a compreensão do processo de propagação da doença em questão. Entretanto, a obtenção dessas relações pode ser difícil, por vezes impossível, de modo que, em muitos trabalhos, se buscam por soluções numéricas. Em alguns casos, porém, os algoritmos empregados na resolução numérica dessas equações apresentam problemas de convergência e de estabilidade, decorrentes da discretização do tempo e do espaço [Johnson, 2009].

Autômatos celulares são convenientes quando se pretende explorar alguma característica espacial do sistema analisado. Em particular, no caso de estudos epidemiológicos, o reticulado do autômato representa os indivíduos e/ou a região onde eles vivem, de modo que a dimensão espacial do sistema é naturalmente levada em consideração. Ainda, sua evolução temporal se dá por meio de regras, de modo que na simulação numérica de um autômato celular não há problemas de estabilidade ou de convergência, como pode ocorrer quando se integram numericamente equações diferenciais.

Há diversos estudos epidemiológicos baseados em autômato celular [Ahmed et al., 1998; Fuentes and Kuperman, 1999; Doran and Laffan, 2005; Slimi et al., 2009; Silva and Monteiro, 2014; Yang et al., 2015]. Aqui, usa-se um modelo do tipo SIR (suscetível-infectado-removido) [Kermack and McKendrick, 1927] formulado em termos de regras de um autômato celular probabilista. $\mathrm{O}$ objetivo é investigar como diferentes distribuições espaciais do vetor afetam a evolução temporal da doença; em particular, como afetam o número de pessoas infectadas em regime permanente (isto é, depois que o sistema atingiu seu atrator).

Este trabalho está organizado da seguinte maneira: na Seção 2, descreve-se o modelo epidemiológico; na Seção 3, apresentam-se os resultados obtidos em simulações computacionais; e, na Seção 4, discutem-se esses resultados.

\section{O Modelo}

Considere que a população hospedeira seja representada por um reticulado (uma matriz) de dimensões são $n \times n$, de modo que cada célula do reticulado corresponde a um único indivíduo. Assuma que cada célula tenha oito vizinhas, que são aquelas localizadas em seu entorno. A fim de que todas as células tenham o mesmo número de vizinhas, impõese que a primeira linha do reticulado está em contato com $n$-ésima linha, e que a primeira coluna está em contato com a $n$-ésima coluna, de modo que a superfície assim formada possui um formato toroidal. Dessa maneira, evitam-se efeitos de borda. Suponha, ainda, que a quantidade de mosquitos associada à $j$-ésima célula seja dada por $m_{j}$. Assim, a quantidade total de mosquitos no reticulado vale $\Sigma_{j} m_{j}=M$.

A cada passo de tempo $t$, cada célula está em um de três estados: suscetível $S$, infectado $I$ ou recuperado $R$. Assume-se que as regras de transição de estados, que determinam a evolução temporal desse sistema, são probabilistas. As regras são as seguintes: 
- A cada passo de tempo, a probabilidade $P_{\text {inf }}$ de um indivíduo suscetível se tornar infectado é dada por $P_{i n f}=1-e^{-k v}$, sendo $v$ o número de vizinhos infectados e $k$ a soma dos valores de $m_{j}$ das oito células vizinhas. Portanto, se $v=0$ e/ou $k=0$, então $P_{i n f}=0$; ou seja, se não há doente e/ou mosquito na vizinhança de um indivíduo suscetível no instante $t$, é nula a probabilidade de ele se tornar infectado no instante $t+1$. Observe que quanto maior o valor de $v$ e/ou $k$, maior o valor de $P_{\text {inf }}$ (de fato, se $k v \rightarrow \infty$, então $P_{\text {inf }} \rightarrow 1$ ).

- A cada passo de tempo, a probabilidade de um indivíduo infectado se curar é dada por $P_{\text {cura }}$. Suponha que a cura confira imunidade permanente em relação ao patógeno em questão.

- A cada passo de tempo, a probabilidade de um infectado morrer vale $P_{\text {morte-I. }}$.

- A cada passo de tempo, um indivíduo recuperado (curado) pode morrer com probabilidade $P_{\text {morte-R }}$.

Por simplicidade, supõe-se que, quando um indivíduo $I$ ou $R$ morre, nasce um indivíduo $S$ em seu lugar. Dessa maneira, o número total de indivíduos da população permanece constante e igual a $N=n^{2}$. Essa hipótese é válida em populações em que o número de mortes é compensado pelo número de nascimentos.

Observe que os parâmetros do modelo são: o número total de indivíduos $N$, as probabilidades $P_{\text {cura }}, P_{\text {morte-I }}$ e $P_{\text {morte-R }}$, a quantidade total de mosquitos $M$ e a distribuição espacial $m_{j}$ dos mosquitos. Esse modelo pode ser adequado para investigar a propagação, por exemplo, de chikungunya, cuja cura confere imunidade de longo prazo. Com as devidas adaptações, esse modelo por ser usado para estudar outras doenças transmitidas por mosquitos. Por exemplo, para se estudar a propagação de dengue, devem-se levar em conta seus quatro sorotipos (ou seja, o grupo dos indivíduos infectados e o grupo dos indivíduos recuperados devem ser divididos em subgrupos). Para se estudar a disseminação do vírus que causa zika, talvez se deva considerar também a possibilidade de transmissão direta via contato sexual [Ministério da Saúde, 2017a].

Os estados de todas as células são atualizados simultaneamente, a cada passo de tempo, o que é comumente chamado de atualização síncrona. Um modelo similar a esse, com $k$ constante, foi proposto por Schimit and Monteiro (2009).

Na próxima seção, apresentam-se os resultados obtidos em simulações com esse autômato celular probabilista.

\section{Simulações Numéricas}

Aqui, tomam-se $n=200$ (assim, a população hospedeira é formada de $N=40000$ indivíduos), $P_{\text {cura }}=60 \%, P_{\text {morte-I }}=30 \%$ e $P_{\text {morte- } R}=10 \%$. Em todas as simulações, a condição inicial é $S(0) / N=99 \%, I(0) / N=1 \%$ e $R(0) / N=0 \%$ (portanto, em $t=0,99 \%$ da população é suscetível ao patógeno e $1 \%$ está infectada). Outros valores de parâmetros e de condições iniciais foram simulados, mas os resultados são qualitativamente iguais aos relatados a seguir. 
Consideram-se quatro distribuições espaciais do vetor:

- Todas as células têm um mesmo valor não nulo de $m_{j}=m_{\text {uniforme. }}$. Essa distribuição é denominada de uniforme, pois todas as células possuem uma mesma quantidade de mosquitos.

- Cada célula tem $50 \%$ de chance de ter um mesmo valor não nulo de $m_{j}=m_{\text {aleatória }}$ e $50 \%$ de chance de não ter mosquito. Essa distribuição é chamada de aleatória.

- Todas as células das colunas pares têm $m_{j}=m_{\text {coluna }}$ não nulo; todas as células das colunas ímpares têm $m_{j}$ igual a zero. Chama-se esse caso de distribuição coluna.

- As células que formam uma matriz $p \times p$ no interior do reticulado (isto é, $p<n$ ) possuem $m_{j}=m_{\text {concentrada }}$ não nulo; para as demais células (aquelas fora dessa matriz $p \times p), m_{j}$ vale zero. Essa distribuição é chamada de concentrada.

Nessas distribuições, $m_{\text {uniforme }}, m_{\text {aleatória }}, m_{\text {coluna }}$ e $m_{\text {concentrada }}$ são constantes. Neste trabalho, investiga-se como essas quatro distribuições afetam a propagação da doença, impondo que o valor de $M$ é o mesmo para as quatro distribuições. Assim, tem-se a mesma quantidade total de mosquitos nos quatro cenários. Por exemplo, $M=4000$ equivale a $m_{\text {uniforme }}=0,1 ; m_{\text {aleatória }}=m_{\text {coluna }}=0,2$ e $m_{\text {concentrada }}=0,4$ para $p=100$ (neste último caso, os mosquitos vivem numa matriz de dimensões $100 \times 100$ contida na matriz $200 \times 200)$. Já $M=12000$ corresponde a $m_{\text {uniforme }}=0,3 ; \quad m_{\text {aleatória }}=m_{\text {coluna }}=0,6 \quad \mathrm{e}$ $m_{\text {concentrada }}=1,2$ (para $\left.p=100\right) ; M=20000$ corresponde a $m_{\text {uniforme }}=0,5 ; m_{\text {aleatória }}=m_{\text {coluna }}=1$ e $m_{\text {concentrada }}=2$ (para $p=100$ ) e $M=120000$ implica $m_{\text {uniforme }}=3 ; m_{\text {aleatória }}=m_{\text {coluna }}=6$ e $m_{\text {concentrada }}=12($ para $p=100)$.

A Tabela 1 apresenta as médias das quantidades normalizadas de infectados em regime permanente, em função das quatro distribuições espaciais consideradas e de quatro valores de $M$. Para cada distribuição espacial, foram feitas 10 simulações. Para cada simulação, calculou-se o valor médio de $I(t) / N$ nos últimos 50 passos de tempo. Os números apresentados na Tabela 1 são as médias (considerando as 10 simulações) desses valores médios de $I(t) / N$ (considerando os últimos 50 passos de tempo de cada simulação) e os respectivos desvios padrões.

Tabela 1. Médias de $I(t) / N$ considerando os últimos 50 passos de tempo de 10 simulações, em função das distribuições espaciais e de $M$.

\begin{tabular}{|c|c|c|c|c|}
\hline & $\begin{array}{c}\text { distribuição } \\
\text { uniforme }\end{array}$ & $\begin{array}{c}\text { distribuição } \\
\text { aleatória }\end{array}$ & $\begin{array}{c}\text { distribuição } \\
\text { coluna }\end{array}$ & $\begin{array}{c}\text { distribuição } \\
\text { concentrada }\end{array}$ \\
\hline$M=4000$ & $0,1022 \pm 0,0004$ & $0,0984 \pm 0,0005$ & $0,0963 \pm 0,0003$ & $0,0299 \pm 0,0002$ \\
\hline$M=12000$ & $0,1149 \pm 0,0003$ & $0,1131 \pm 0,0005$ & $0,1127 \pm 0,0004$ & $0,0301 \pm 0,0002$ \\
\hline$M=20000$ & $0,1164 \pm 0,0003$ & $0,1153 \pm 0,0004$ & $0,1154 \pm 0,0004$ & $0,0302 \pm 0,0002$ \\
\hline$M=120000$ & $0,1168 \pm 0,0003$ & $0,1163 \pm 0,0003$ & $0,1167 \pm 0,0004$ & $0,0302 \pm 0,0002$ \\
\hline
\end{tabular}


A Figura 1 mostra um exemplo da evolução temporal de $S(t) / N, I(t) / N$ e $R(t) / N$ por 200 passos de tempo, para uma distribuição uniforme de mosquitos com $M=4000$. Note que os infectados são, em regime permanente, cerca de $10 \%$ da população (um valor que obviamente está de acordo com a média correspondente ao caso de distribuição uniforme e $M=4000$ mostrado na Tabela 1).

A Tabela 1 revela que, para todas as distribuições, a quantidade de infectados cresce (lentamente) com o valor de $M$. Esse crescimento é esperado: quanto mais mosquitos, mais infecções devem ocorrer para uma dada distribuição espacial. Essa tabela revela também que as distribuições uniforme, aleatória e coluna têm médias próximas (em torno de 10\%), mas a distribuição concentrada claramente leva a um menor número de infectados (em torno de $3 \%$ ).

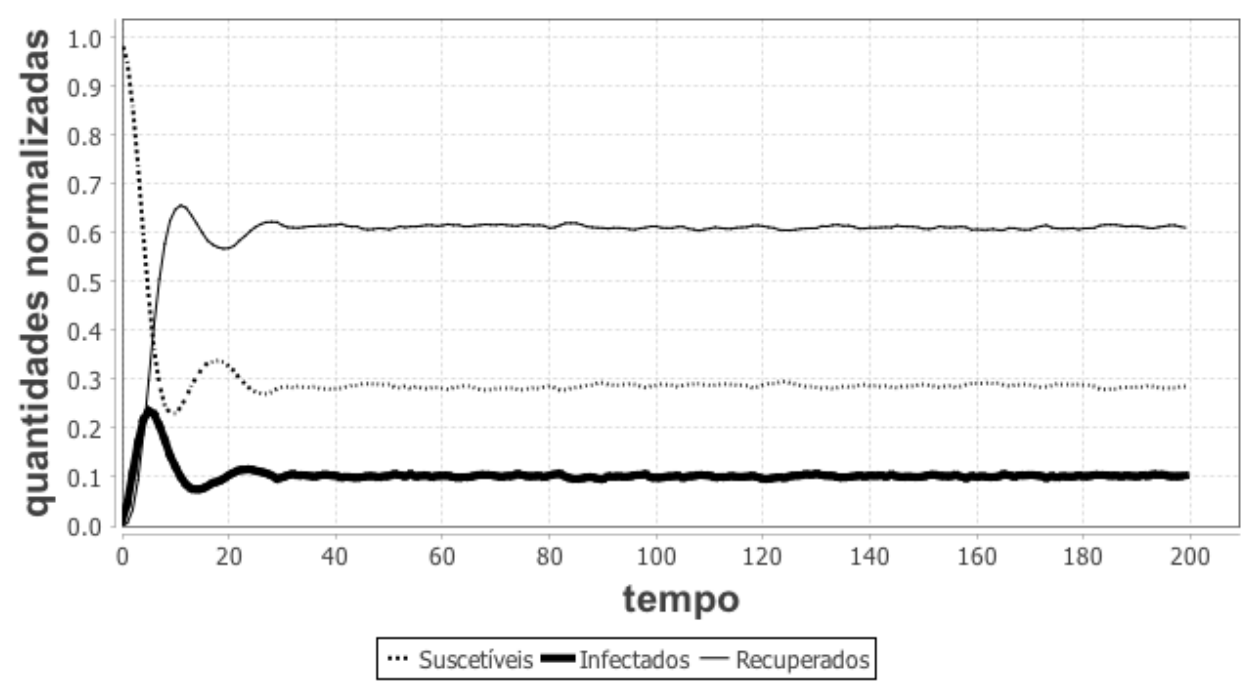

Figura 1. Exemplo de evolução temporal de $S(t) / N, I(t) / N$ e $R(t) / N$. A condição inicial é $S(0) / N=99 \%, I(0) / N=1 \%$ e $R(0) / N=0 \%$. Valores dos parâmetros do $A C P$ : $n=200(N=40000), P_{\text {cura }}=60 \%, P_{\text {morte-l }}=30 \%, P_{\text {morte- } R}=10 \%$, distribuição de vetores uniforme com $m_{\text {uniforme }}=0,1(M=4000)$.

Um trabalho teórico seminal sobre doenças transmitidas por vetores foi realizado por Ross (1908). Nesse trabalho, Ross apresentou o teorema do mosquito, no qual ele demonstra que a malária seria naturalmente erradicada de uma dada região se a quantidade de mosquitos nessa região ficasse abaixo de um valor crítico (Ross, 1908; Fine 1975). Esse trabalho, entretanto, não considera a dimensão espacial.

Inspirado nesse teorema, foram realizadas outras simulações para cada uma das quatro distribuições de vetores já descritas. $\mathrm{O}$ objetivo dessas simulações é determinar o valor de $M$ abaixo do qual a doença não se torna endêmica em 10 simulações consecutivas; ou seja, o valor de $M$ abaixo do qual o patógeno é naturalmente erradicado da população hospedeira em 10 simulações consecutivas. Obtiveram-se os seguintes valores: 1200 para a distribuição uniforme, 1000 para as distribuições aleatória e coluna, 
e 300 para a distribuição concentrada. Esse resultado é bastante interessante. Apesar de a distribuição concentrada causar um menor número médio de infectados, essa distribuição é aquela que mantém a doença endêmica na população com uma menor quantidade de mosquitos. Por exemplo, se há 500 mosquitos no reticulado, a doença desaparece com o passar do tempo (ou seja, $I(t) \rightarrow 0$ ) se os mosquitos estão distribuídos de maneira uniforme, aleatória ou por coluna, mas ela permanece se os mosquitos ficam concentrados numa dada região do reticulado.

\section{Conclusões}

Neste trabalho, propôs-se um modelo epidemiológico do tipo SIR para investigar como diferentes distribuições espaciais do vetor afetam a propagação de doenças por ele transmitidas. O modelo baseia-se num autômato celular probabilista, que é caracterizado pela geometria e pelas dimensões do seu reticulado, pelos estados possíveis, pela vizinhança de cada célula, pelas regras que governam as transições de estados, e pela maneira com que essas regras são aplicadas (com o passar do tempo) às células que compõem o reticulado. Aqui, o autômato possui um reticulado bidimensional com condição de contorno periódica, com cada célula, a cada instante, assumindo um de três estados e tendo como vizinhança as oito células ao seu redor. A evolução temporal dos estados das células se dá pela aplicação de regras probabilistas de maneira síncrona. Foram consideradas quatro maneiras de distribuir o vetor pelo reticulado.

As simulações numéricas mostraram que, para uma mesma quantidade $M$ de vetores, o número de infectados é aproximadamente o mesmo para as distribuições uniforme, aleatória e coluna; já, para a distribuição concentrada, esse número é claramente menor. Entretanto, a distribuição concentrada é aquela que exige um menor valor de $M$ para que a doença seja eliminada da população hospedeira. Portanto, este trabalho sugere que é fundamental eliminar os locais de grande concentração de vetores, pois esses podem ser os principais responsáveis por manter a doença endêmica na população.

Pretende-se dar continuidade a este trabalho considerando outras distribuições espaciais do vetor e que essas distribuições podem se modificar com o passar do tempo (em função, por exemplo, da variação da temperatura e da quantidade de chuvas).

\section{Agradecimentos}

LHAM agradece o suporte financeiro do CNPq.

\section{Referências}

Ahmed, E., Agiza, H. N., and Hassan, S. Z. (1998), "On modeling Hepatitis B transmission using cellular automata", Journal of Statistical Physics, v. 92, n. 3, p. 707-712.

Anderson, R. M. and May, R. M. (1991), Infectious Diseases of Humans, Oxford University Press.

Doran, J. R. and Laffan, S. W. (2005), "Simulating the spatial dynamics of foot and mouth disease outbreaks in feral pigs and livestock in Queensland, Australia, using a 
susceptible-infected-recovered cellular automata model", Preventive Veterinary Medicine, v. 70, n. 1-2, 133-152.

Fine, P. E. (1975), "Ross's a priori pathometry - a perspective", Proceedings of the Royal Society of Medicine (London), v. 68, n. 9, p.547-551.

Friis, R. H. (2009), Epidemiology 101, Jones \& Bartlett Learning.

Fuentes, M. A. and Kuperman, M. N. (1999), "Cellular automata and epidemiological models with spatial dependence”, Physica A, v. 267, n. 3-4, p. 471-486.

Hethcote, H. W. (2000), "The mathematics of infectious diseases", SIAM Review, v. 42 , n. 4 , p. $599-653$.

Johnson, C. (2009), Numerical Solution of Partial Differential Equations by the Finite Element Method, Dover.

Kermack, W. O. and McKendrick, A. G. (1927), "A contribution to the mathematical theory of epidemics", Proceedings of the Royal Society A, v. 115, n. 772, p. 700-721.

Ministério da Saúde (2017a), "Prevenção e Combate: Dengue, Chikungunya e Zika" http://combateaedes.saude.gov.br/pt/tira-duvidas, acessado em março de 2017.

Ministério da Saúde (2017b), "Monitoramento dos Casos de Dengue, Febre de Chikungunya e Febre pelo Vírus Zika até a Semana Epidemiológica 4, 2017”, Boletim Epidemiológico, v. 48, n. 5, p. 1-9.

Ministério da Saúde (2017c), "Monitoramento dos Casos e Óbitos de Febre Amarela no Brasil", Informe n $23 / 2017$, p. 1-5.

Murray, J. D. (2011), Mathematical Biology II: Spatial Models and Biomedical Applications, Springer, $3^{\text {rd }}$ edition.

Ross, R. (1908), Report on the Prevention of Malaria in Mauritius, Waterlow and Sons.

Schimit, P. H. T. and Monteiro, L. H. A. (2009), "On the basic reproduction number and the topological properties of the contact network: An epidemiological study in mainly locally connected cellular automata”. Ecological Modelling, v. 220, n. 7, p. 1034-1042.

Silva, H. A. L. R. and Monteiro, L. H. A. (2014), "Self-sustained oscillations in epidemic models with infective immigrants", Ecological Complexity, v. 17, p. 40-45.

Slimi, R., El Yacoubi, S., Dumonteil, E., and Gourbiere, S. (2009), "A cellular automata model for Chagas disease", Applied Mathematical Modelling, v. 33, n. 2, p. 10721085 .

Wolfram, S. (1994). Cellular Automata and Complexity: Collected Papers, Westview Press.

Yang, F., Yang, Q., Liu, X., and Wang, P. (2015), "SIS evolutionary game model and multi-agent simulation of an infectious disease emergency", Technology and Health Care, v. 23, n. s2, p. S603-S613. 